\title{
Número de horas de cuidados de enfermagem em Unidade de Terapia Intensiva de Adultos
}

\author{
NUMBER OF NURSING CARE HOURS IN AN INTENSIVE CARE UNIT \\ NÚMERO DE HORAS DE CUIDADOS DE ENFERMERÍA EN UNIDAD DE TERAPIA INTENSIVA
}

\author{
Ana Maria Tranquitelli ${ }^{1}$, Maria Helena Trench Ciampone ${ }^{2}$
}

\section{RESUMO}

$\mathrm{O}$ adequado dimensionamento de pessoal de enfermagem nas Terapias Intensivas tem sido uma grande preocupação para os enfermeiros que nelas atuam a despeito dos diferentes modelos de classificação dos pacientes propostos, no âmbito nacional e internacional. No presente estudo de caso, os objetivos foram estabelecer o tempo médio despendido na assistência direta de enfermagem em uma Unidade de Terapia Intensiva geral e calcular o número médio de horas de assistência direta de enfermagem prestada a esses pacientes. Para tanto, primeiramente, classificaram-se os procedimentos de enfermagem desenvolvidos com pacientes da UTI, de acordo com a sua complexidade: baixa, média e alta. A seguir, cronometrou-se o tempo médio despendido na realização desses procedimentos, a fim de se encontrar o tempo médio de assistência direta de enfermagem prestada aos pacientes, durante um período de três meses consecutivos.

\section{DESCRITORES}

Unidades de Terapia Intensiva. Pacientes/classificação.

Recursos humanos de enfermagem no hospital. Cuidados de enfermagem/ organização \& administração. Carga de trabalho.

\section{ABSTRACT}

The adequate sizing of the nursing staff in Intensive Care Units has been one of the main concerns for the nurses that work in them, in spite of different classification models for patients that have been proposed at the domestic and international levels. In the present case study, the goals were to establish the average time spent in direct nursing assistance in an ICU and to calculate the average number of hours of direct nursing assistance delivered to its patients. In order to do that, the first step was to classify the nursing procedures carried out with ICU patients according to their complexity: low, medium and high. Next, the average time spent in theses procedures was counted during a three-month period, in order to find out the average time of direct nursing assistance delivered to patients.

\section{KEY WORDS}

Intensive Care Units.

Patients/classification.

Nursing staff, hospital.

Nursing care/organization \& administration.

Workload.

\section{RESUMEN}

La dimensión adecuada del personal de enfermería en los centros de terapias intensivas ha sido una gran preocupación para los enfermeros que actúan en ellos, llevando en consideración los diferentes modelos de clasificación de los pacientes propuestos, tanto en el ámbito nacional como en el internacional. En este estudio, los objetivos fueron establecer el tiempo medio que se utilizó en la asistencia directa de enfermería en un centro de terapia intensiva (CTI) general y calcular el número medio de horas de asistencia directa dispendidas con estos pacientes. Para eso fueron clasificados primeramente los procesos de enfermería desarrollados con pacientes de CTI, de acuerdo con su complejidad: baja, media y alta. Enseguida se cronometró el tiempo medio que se gastó en la realización de estos procedimientos con la finalidad de encontrar el tiempo medio de la atención de enfermería dedicada a los pacientes, durante un periodo de tiempo de tres meses consecutivos.

\section{DESCRIPTORES}

Unidades de Terapia Intensiva.

Pacientes/clasificación.

Personal de enfermería

en hospital.

Cuidados de enfermería/ organización \& administración. Carga de trabajo.

\author{
1 Enfermeira. Doutora \\ pela Escola de \\ Enfermagem da \\ Universidade de \\ São Paulo (EEUSP). \\ São Paulo, SP, Brasil. \\ anatranquitelli@ \\ telefonica.com.br \\ 2 Enfermeira. Profes- \\ sora Titular do Depar- \\ tamento de Orienta- \\ ção Profissional da \\ ĖEUSP.São Paulo, \\ SP, Brasil. \\ mhciamp@usp.br
}




\section{INTRODUÇÃO}

As Unidades de Terapia Intensiva (UTIs) surgiram no Brasil na década de 70 do século XX, encontrando campo fértil no país no auge do milagre econômico que, no contexto, privilegiava um modelo econômico concentrador de renda e uma política voltada à modernização e ao desenvolvimento, o que repercutiu no setor da Saúde em que a expansão se deu às custas da ação do Estado ${ }^{(1)}$.

O perfil do sistema de saúde, a partir desse período, caracterizou-se pela absorção de avanços tecnológicos oriundos do primeiro mundo, que possibilitaram o aprimoramento dos métodos diagnósticos e terapêuticos pela absorção de recursos altamente sofisticados que privilegiam a assistência nos níveis de atenção secundário e terciário, sobrepujando a necessidade de atenção primária ${ }^{(2)}$.

Conceitualmente, verifica-se que a Unidade de Terapia Intensiva destina-se ao atendimento de pacientes em estado agudo ou crítico, mas recuperável, que requerem assistência médica e de enfermagem permanente e especializada. São pacientes sujeitos à instabilidade de funções vitais, que necessitam do apoio de equipamentos especiais de diagnóstico e tratamento ${ }^{(3)}$.

Portanto, com o surgimento das UTI's, pacientes graves que, antes do advento destas unidades, tinham pouca ou nenhuma chance de sobrevivência, passaram a utilizar recursos de que até então não dispunham.

De acordo com o enfoque assistencial voltado aos problemas no âmbito individual, pressupõe-se que as unidades de cuidados devam ser adequadas às necessidades da clientela atendida. Assim, devem ser providas adequadamente, em sua estrutura física, de recursos humanos e de recursos materiais, constituindo-se em suporte para implantação de uma assistência efetiva ao paciente hospitalizado, principalmente nas UTI's, em função da sua especificidade.

Os estudos relacionados aos métodos de dimensionamento de pessoal na área da enfermagem têm demonstrado que os diferentes graus de complexidade assistencial dos pacientes, em unidades de internação passaram a ser considerados como parâmetro importante para prever e prover recursos humanos ${ }^{(4-7)}$.

Assim, tendo por base estudos que comprovavam a otimização do tempo de assistência de enfermagem quando os pacientes ficavam agrupados de acordo com a complexidade de cuidados necessários, diversas instituições hospitalares começaram a utilizar o conceito de Sistema de Classificação de Pacientes (SCP) e a implantar a forma de organizar e dividir o trabalho denominada de Cuidado Progressivo do Paciente (CPP) ${ }^{(8-11)}$.
Esse conceito, na prática gerencial do enfermeiro, orienta o modo de dividir e organizar o trabalho nas unidades e tem por finalidade básica adequar as respectivas unidades de cuidados às necessidades assistenciais dos pacientes, de acordo com o grau de dependência destes em relação à assistência $^{(12-13)}$.

A partir da década de 80, é possível afirmar que, nas instituições hospitalares, houve a tendência e preocupação em alocar o paciente certo na unidade certa, onde se pretende que ele possa dispor de uma infra-estrutura organizada de tal maneira, que todas suas necessidades sejam atendidas com qualidade.

Embora no Brasil, a noção de classificação de pacientes tenha sido adotada mais tardiamente, na década de 80, esta difundiu-se a partir do trabalho realizado pela Escola de Enfermagem de Pittisburg em 1950, tendo como finalidade determinar as necessidades de cuidados de enfermagem para pacientes com diagnósticos de clinica médica e cirúrgica ${ }^{(14)}$.

Ainda a referida autora afirma que um dos parâmetros vinculados ao Sistema de Classificação de Pacientes (SPC) é o número médio de horas despendidas pela equipe de enfermagem, segundo cada categoria de paciente da classificação. Contudo, os estudos nessa área têm mostrado que esses parâmetros diferem de um modelo de classificação para outro e nesses, nem sempre estão explicitadas as condições e o método como o mesmo foi definido.

Segundo a resolução COFEN - 189/96, que estabelece parâmetros para o dimensionamento do quadro de profissionais de enfermagem nas Instituições de Saúde, compete ao enfermeiro estabelecer o quadro quanti-qualitativo de profissionais necessários à prestação da assistência de enfermagem ${ }^{(5)}$.

Para a assistência intensiva, o Conselho Federal de Enfermagem (COFEN) em 1996, estabeleceu 15,4 horas de enfermagem/dia por cliente de assistência intensiva. No entanto, percebe-se que esses valores numéricos, por mais expressivos que sejam, nem sempre refletem de forma efetiva a dinâmica da assistência de enfermagem na UTI, principalmente ao se considerar as especificidades de cada local.

Nas unidades chefiadas por uma das pesquisadoras deste estudo, avaliou-se que as necessidades relativas ao dimensionamento de pessoal não estavam sendo atendidas aplicando-se apenas o parâmetro das horas de assistência de enfermagem estabelecidas na Resolução 189/96, o que despertou dúvidas e estimulou o interesse para o desenvolvimento do estudo em questão.

Este fato corroborou a necessidade de realizar uma pesquisa que possibilitasse identificar e analisar a especificidade das horas de assistência direta de enfermagem na UTI campo de estudo, o que permitiria um dimensionamento de pessoal mais adequado à realidade local. 
Isso posto, a finalidade do presente estudo foi de fornecer subsídios para definir o número de horas de cuidados de enfermagem, de modo a propiciar a organização e distribuição do trabalho, fundamentado no sistema de classificação dos pacientes de acordo com o grau de dependência de assistência, na unidade campo de prática de uma das pesquisadoras.

\section{OBJETIVOS}

- Verificar o tempo médio despendido para executar os procedimentos básicos na assistência do paciente crítico em uma UTI geral, com base na observação da realidade.

- Calcular o número médio de horas de assistência de enfermagem prestadas aos pacientes internados nessa UTI.

\section{MÉTODO}

\section{Campo de estudo}

O estudo foi realizado em um hospital geral da rede privada do município de São Paulo. Trata-se de um hospital que dispõe de 258 leitos ativos, acrescido de mais 12 leitos de repouso, que atende todas as especialidades médicas. Como hospital beneficente, destina 20\% do total de leitos aos pacientes do Sistema Único de Saúde.

O número total de funcionários é 2.180, sendo que os funcionários da Enfermagem totalizam 763 (35\%), assim distribuídos por categoria: um gerente de serviço, dois assistentes de gerência, quinze enfermeiras encarregadas, 146 enfermeiras assistenciais, 480 auxiliares de enfermagem, 40 agentes de transporte.

A UTI onde foi realizado este estudo conta com 12 leitos para atendimento de adultos portadores de patologias clínicas e cirúrgicas e 52 profissionais de enfermagem, sendo 01 (1,9\%) enfermeira encarregada, 14 (26,9\%) enfermeiras assistenciais, $32(61,6 \%)$ auxiliares de enfermagem e cinco $(9,6 \%)$ agentes de transporte.

É importante destacar que a jornada de trabalho do pessoal de enfermagem da UTI, nesse cenário, é de 36 horas semanais e os funcionários da jornada noturna fazem 12 por 36 horas de descanso com duas folgas semanais.

\section{Coleta de dados}

A coleta de dados foi realizada na Unidade de Terapia Intensiva em um período de três meses consecutivos. Todos os prontuários com as informações do conjunto de procedimentos realizados com os pacientes internados na unidade, no período, foram incluídos, após aprovação do projeto pelo Comitê de Ética da Instituição.

O estudo foi dividido em duas etapas, na primeira buscou-se estabelecer a média de tempo despendido em cada procedimento desenvolvido pela equipe de enfermagem na UTI. Para se ter validade estatística, os procedimentos realizados na unidade foram agrupados em três categorias: baixa, média e alta complexidade.

Para compor o instrumento de coleta de dados na primeira fase e selecionar os procedimentos realizados com maior freqüência, foram consultados 125 prontuários, correspondentes a todos pacientes internados na UTI no período e listados todos os procedimentos diretamente desenvolvidos pelos profissionais de enfermagem nas 24 horas do dia.

Para cada procedimento classificado pelos enfermeiros por categoria, foram eleitos os cinco procedimentos mais freqüentes na Unidade e, a partir daí destacou-se, para cada um deles, cinco diferentes profissionais executantes com o mesmo grau de formação e tempo médio de trabalho semelhante na UTI. Realizou-se a observação sistemática da execução desses procedimentos de acordo com cada um dos executantes e registrou-se o tempo despendido em cada procedimento, mediante prévia descrição das etapas que marcavam o início e o fim dos mesmos, de acordo com o manual de procedimentos da instituição.

Os procedimentos, considerados pelos enfermeiros da unidade como sendo de baixa complexidade e que eram realizados com maior freqüência e que foram selecionados para computar o tempo médio de execução foram: os controles gerais, alimentação, manuseio de cateteres, higiene corporal e preparo de medicação.

Os procedimentos de média complexidade, que foram eleitos pelo mesmo critério foram: exame físico, fechamento de balanço hídrico, colocação de horário nas prescrições médicas, coleta de sangue e orientação às famílias. Os procedimentos considerados pelos enfermeiros do setor como sendo de alta complexidade e realizados com maior freqüência foram: transporte, admissão, evolução, cuidados com feridas e passagem de plantão.

Para cada profissional executante de cada procedimento, cronometrou-se cinco vezes o desenvolvimento do mesmo. Portanto, houve 25 diferentes mensurações do tempo de cada procedimento realizado para se chegar a uma média. Para a obtenção das três médias finais do tempo de desenvolvimento dos procedimentos de baixa, média e alta complexidade computou-se um total de 375 mensurações. Vale ressaltar que não foram encontradas referências de estudos de tempo e movimento suficientes na área de enfermagem, que subsidiasse o número de observações e mensurações a serem realizadas para encontrar o tempo médio; portanto, os parâmetros que foram estipulados para o presente estudo seguiram a orientação de um estatístico.

A segunda etapa da coleta de dados constou do preenchimento de um segundo instrumento em que, diariamente, para todos os pacientes internados na unidade de terapia 
intensiva, no período estipulado, após serem avaliados segundo a necessidade de realização dos procedimentos previamente definidos, registrada-se o número de vezes que estes procedimentos eram realizados; a média de tempo correspondente à realização individual do procedimento e, em última coluna, o tempo despendido, ao longo das 24 horas, na realização destes, considerando-se quantas vezes foi necessária sua execução.

O instrumento utilizado na segunda fase da coleta possuía um campo de codificação da identificação dos pacientes, para que pudéssemos retornar ao prontuário caso houvesse dúvidas a esclarecer; uma coluna onde eram listados os procedimentos; uma coluna para marcação do número de vezes que o procedimento foi realizado; outra coluna onde foram colocados os valores das médias de tempo encontradas em cada um desses e uma quarta coluna onde constava o total de horas obtidas da multiplicação do número de vezes que cada procedimento foi realizado no paciente com a média de tempo a ele correspondente.

Com o preenchimento desse instrumento, a cada dia obteve-se um total final de horas, minutos e segundos consumidos nas 24 horas/dia, na assistência a cada paciente, no período de tempo demarcado no estudo.

A seguir, esses dados diários foram transpostos para uma planilha construída no Programa Excel, possibilitando que chegássemos a uma média mensal de tempo gasto em cada procedimento das três categorias de complexidade.

\section{APRESENTAÇÃOE DISCUSSÃO DOS RESULTADOS}

Fizeram parte do estudo o conjunto dos procedimentos realizados em 125 pacientes internados em uma unidade de terapia intensiva durante um período de três meses. Desses 125 pacientes, constatamos que $72 \%$ permaneceram internados na UTI de 1 a 4 dias, seguida de $12 \%$ dos pacientes que permaneceram de cinco a oito dias, $5 \%$ ficaram na unidade de nove a doze dias, $3 \%$ de treze a dezesseis, $5 \%$ de dezessete a vinte dias e $3 \%$ mais que vinte e um dias.

Com base na observação da realidade, e pela cronometragem do tempo utilizado para a realização dos procedimentos, foram encontradas as três médias finais para cada categoria de procedimentos que nortearam toda a pesquisa.

A Figura 1 demonstra o tempo médio despendido em procedimentos de baixa complexidade. Dentre os cinco procedimentos eleitos para as 125 cronometragens de tempo, encontrou-se os seguintes tempos: controles gerais (18min01seg); alimentação (5min42seg); manuseio de cateter (9min19seg); higiene corporal (29min20seg) e preparo de medicamentos (5min22seg). Vale ressaltar que, na unidade campo do estudo, estes procedimentos são executados por auxiliares de enfermagem.

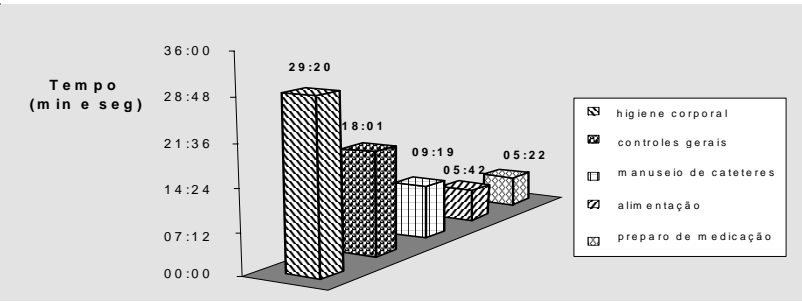

Figura 1 - Tempo médio despendido pelos auxiliares de enfermagem na realização de procedimentos de baixa complexidade na UTI campo de estudo, no período de janeiro a março - São Paulo - 1998

Observando a Figura 1, é importante salientar que no procedimento de higiene corporal correspondeu a maior média de tempo (29min20seg). Cabe salientar que, para o dimensionamento de pessoal, o tempo médio gasto na execução do procedimento deveria ser multiplicado pelo número real de profissionais que costumam ministrar o cuidado, isto é, se no cotidiano de cada unidade a higiene corporal costuma ser feita por dois executantes, esse dado deveria ser computado.

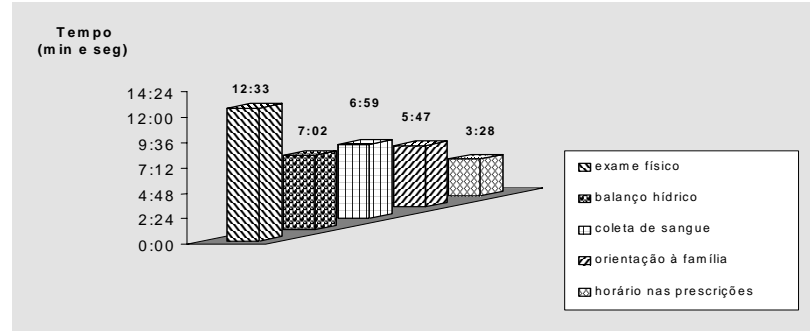

Figura 2 - Tempo médio despendido pelas enfermeiras na realização de procedimentos de média complexidade, na UTI campo de estudo, de janeiro a março - São Paulo 1998

A Figura 2 demonstra o tempo médio despendido em procedimentos de média complexidade. Dentre os cinco procedimentos eleitos para as 125 medidas foram encontrados os seguintes tempos: exame físico (12min33seg), fechamento de balanço hídrico (7min02seg), horário nas prescrições (3min28seg), coleta de sangue (6min59seg) e orientação à família (5min47seg). Os procedimentos foram cronometrados tendo como executantes as enfermeiras assistenciais.

Dado que as horas de assistência foram obtidas pela cronometragem do tempo despendido, também nas orientações formais (admissões, altas e horários de visitas) ficou evidente que o tempo contínuo de interação família e equipe não é passível de mensuração direta. Cabe, contudo considerá-lo como parte importante das horas de assistência. Nossa experiência comprova que na prática, essas atividades consomem bastante tempo da equipe de enfermagem, e nem sempre são previsíveis, pois implicam em interação orientada para necessidades específicas em cada caso. 


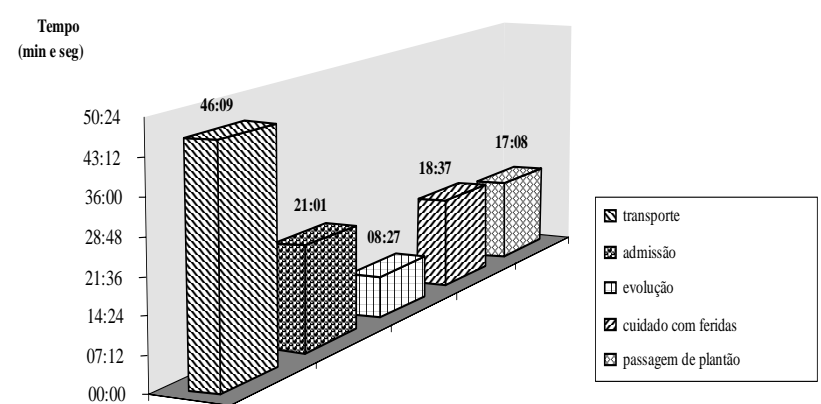

Figura 3 - Tempo médio despendido na realização de procedimentos de alta complexidade, na UTI campo de estudo, de janeiro a março - São Paulo - 1998

A Figura 3 demonstra o tempo médio despendido em procedimentos de alta complexidade, como transporte (46min09seg), admissão (21min01seg), evolução (8min 27seg), cuidados com feridas (18min37seg) e passagem de plantão (27min08seg). Estes cuidados também são prestados por enfermeiras sendo alguns apoiados pelo auxiliar de enfermagem.

Do mesmo modo que a higiene corporal, o transporte nunca é executado por uma única pessoa. Vale ressaltar que, no número de horas de assistência, computou-se apenas o tempo despendido por um profissional, cabendo redimensioná-lo para o cálculo de pessoal em função do número de profissionais realmente envolvidos.

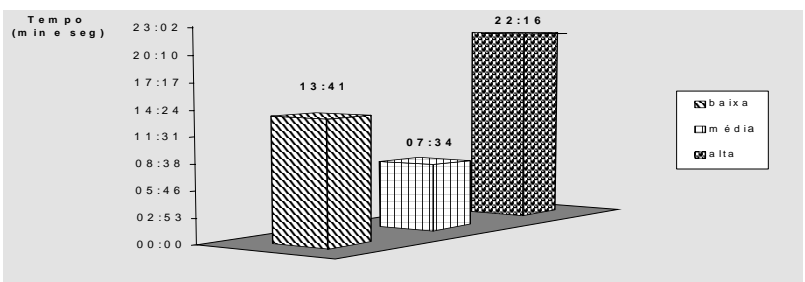

Figura 4 - Tempo médio despendido por procedimento conforme sua complexidade, na UTI campo de estudo, de janeiro a março - São Paulo - 1998

As médias finais encontradas nas 375 mensurações realizadas, que nortearam todo o estudo, estão demonstradas na Figura 4, compreendendo: procedimentos de baixa complexidade (13min41seg), de média complexidade (7min34seg) e de alta complexidade (22min16seg). Vale ressaltar que as médias obtidas nos procedimentos de baixa e média complexidade mostram claramente que o grau de dificuldade na realização dos mesmos não mantêm relação direta com o grau de complexidade, ou seja, procedimentos de baixa complexidade, dado o estado do paciente podem consumir maior tempo que os de maior complexidade.

A partir da obtenção das médias de tempo, foi efetuado um levantamento dos prontuários de todos os pacientes admitidos num período de três meses na UTI em estudo.
Nesses prontuários foram computados, através da anotação relativa ao planejamento de cuidados de enfermagem, evolução de enfermagem e prescrição médica, o número de vezes que os procedimentos selecionados ocorreram nas 24 horas do dia.

Tendo em vista os parâmetros obtidos, no momento em que os procedimentos foram realizados, estes passaram a ser agrupados de acordo com sua complexidade e foram dotados da média de tempo correspondente à sua classificação, sendo assim registrados, de acordo com o número de vezes que eram realizados.

Os dados obtidos permitiram visualizar o número médio de horas de assistência de enfermagem, por dia, por paciente na UTI em estudo no período estipulado. Observou-se, pela disposição dos dados no gráfico de dispersão, uma concentração, nos três meses, de horas de cuidados que variaram de 9 a 13 horas por dia, por paciente, como se vê na Figura 5.

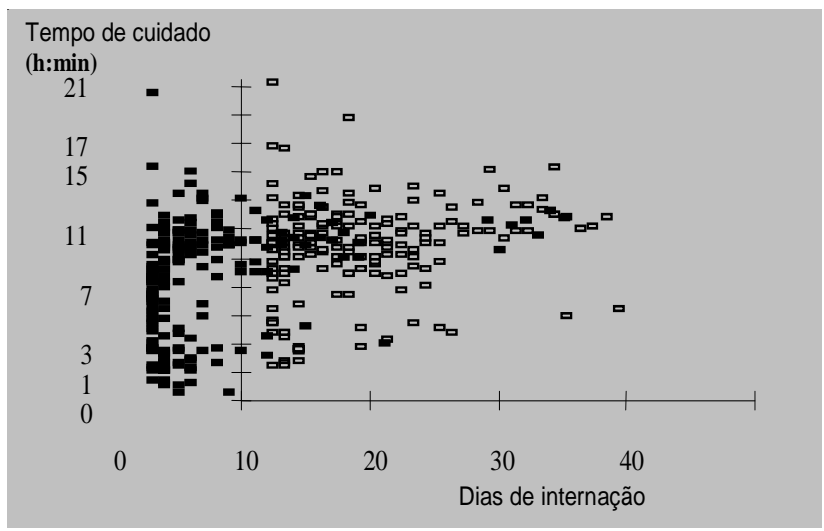

Figura 5 - Número de horas de cuidados de enfermagem, por paciente, por dia de internação, nos meses de janeiro a março - São Paulo - 1998

Esta figura demonstra que a média de permanência dos pacientes na UTI foi de um a quatro dias; e uma concentração mais atenuada de pacientes com média de horas de cuidados abaixo de 9 horas/dia. Isto porque as admissões de pacientes na unidade não têm hora para acontecer, assim como as altas e os óbitos; portanto, a partir das 7 horas da manhã, se padronizou computar o primeiro dia de internação, embora nem sempre neste horário o paciente tenha completado 24 horas na unidade.

A Figura 6 permite apreciar o tempo médio de assistência despendido por paciente por dia de internação, descontados o primeiro e último dia de internação, pelo fato desses dias nem sempre corresponderem ao período completo de 24 horas de assistência. Assim, observa-se novamente uma concentração de horas de cuidados de enfermagem que varia entre 9 e 13 horas. 


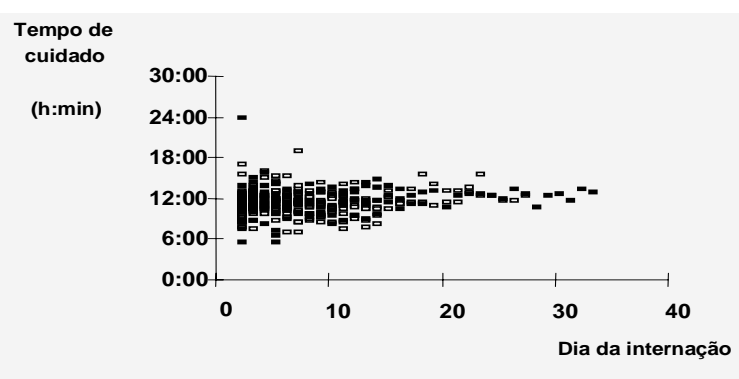

Figura 6 - Número de horas despendidas por paciente, por dia de internação, descontados o primeiro e último dia de internação, nos meses de janeiro a março - São Paulo - 1998

Para melhor compreensão geral dos dados obtidos, verifica-se na Tabela 1 a distribuição das 636 mensurações de horas/dia, de cuidados diretos de enfermagem, na unidade em estudo, de acordo com intervalos que corresponderam ao total de procedimentos mensurados em cada porcentual de horas de assistência.

Tabela 1 - Distribuição do total de medidas de horas de cuidados de enfermagem na UTI campo de estudo de janeiro a março - São Paulo - 1998

\begin{tabular}{lcc}
\hline HORAS (h) & $\begin{array}{c}\text { Número de } \\
\text { Mensurações }\end{array}$ & $\begin{array}{c}\text { Porcentagem } \\
\text { (\%) }\end{array}$ \\
\hline 1:00 a 3:59 & 66 & 10,38 \\
4:00 a 6:59 & 92 & 14,47 \\
7:00 a 9:59 & 131 & 20,60 \\
10:00 a 12:59 & 271 & 42,61 \\
13:00 a 15:59 & 68 & 10,69 \\
16 ou mais & 8 & 1,26 \\
\hline TOTAL & $\mathbf{6 3 6}$ & $\mathbf{1 0 0 , 0 0}$ \\
\hline
\end{tabular}

A Tabela 1 comprova que a maior concentração de mensurações $(42,61 \%)$ ficou no intervalo de consumo de tempo entre 10 horas a $12 \mathrm{~h}$ e $59 \mathrm{~min}$.

A seguir encontrou-se que, dos 20,60\% de mensurações, ou seja, 131 do total de 636 mensurações ficaram no intervalo de consumo de tempo entre $7 \mathrm{~h}$ a $9 \mathrm{~h} 59 \mathrm{~min}$. A somatória desses dois intervalos de tempo consumido corresponde a 64\% das mensurações. Esses dados da Tabela 1 podem ser melhor visualizados na Figura 7.

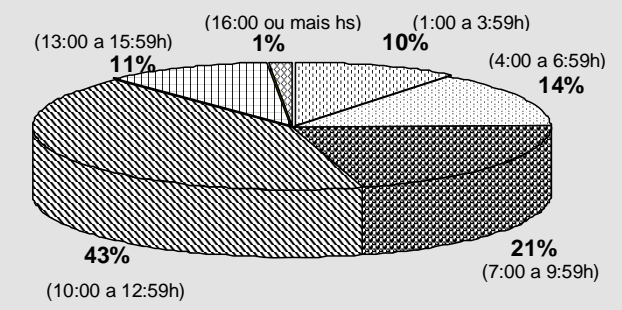

Figura 7 - Distribuição do total das medidas de horas de cuidados diretos por intervalo de tempo, durante o período de janeiro a março - São Paulo - 1998
Assim sendo, considerando o conjunto dos dados obtidos no estudo, estes permitem afirmar que efetivamente o maior quantitativo de horas de cuidados de enfermagem por paciente/dia ficou concentrado no intervalo de 9 a 13 horas.

\section{CONCLUSÃO}

Considerando-se que os objetivos do presente estudo foram verificar o tempo médio despendido para executar os procedimentos básicos na assistência direta do paciente crítico com base na observação da realidade e calcular o número médio de horas de assistência direta de enfermagem prestadas aos pacientes internados nessa UTI, pode-se afirmar que os dados obtidos permitiram o alcance dos mesmos.

Consideramos que a metodologia utilizada de classificação dos procedimentos em três categorias: baixa, média e alta complexidade, mostrou-se útil para o cálculo do número de horas de cuidado direto neste local, bem como poderia ser utilizada em outras unidades, uma vez que permite a seleção dos procedimentos, de acordo com a especificidade da assistência prestada.

Apesar de extremanente trabalhoso, concluímos que somente através de observação sistematizada da realidade é possivel chegar ao tempo médio despendido na assistência de enfermagem para executar os procedimentos básicos ligados à assistência do paciente.

O cálculo do número de horas de assistência de enfermagem, em unidades em que o sistema de classificação de pacientes esteja implantado, evidencia-se como importante ferramenta de gerenciamento da assistência, além de fator essencial para o dimensionamento mais adequado do pessoal de enfermagem.

O registro preciso do tempo despendido permitiu chegar ao número médio de horas de assistência direta de enfermagem prestadas aos pacientes internados na UTI campo de estudo, durante um período de três meses.

As Resoluções COFEN-189/96 e COFEN 293/2004, que fixam e estabelecem parâmetros para o dimensionamento do quadro de profissionais de enfermagem nas unidades assistenciais preconizam respectivamente, 3,0 e 3,8 horas de enfermagem por pacientes de cuidado mínimo ou auto-cuidado; 4 h e 54 min. e 5,6 horas na assistência intermediária; 8,5 horas e 9,4 horas na assistência semi-intensiva e 15,4 horas e 17,9 horas na assistência intensiva, não explicitam as metodologias utilizadas para se chegar a esses índices ${ }^{(5-6)}$.

Já a presente investigação oferece uma metodologia que pode ser replicada em outros estudos para se chegar ao número de horas de cuidados diretos de enfermagem, por paciente/dia, com maior precisão em cada unidade com suas especificidades. 
Embora os resultados a que se chegou quanto ao número de horas de cuidado, na UTI campo de estudo, no período pesquisado, tenha se concentrado no intervalo entre 9 a 13 horas, consideramos que estas não são conclusivas do total de horas de assistência. Um estudo mais aprofundado das horas de cuidado direto e indireto, provavelmente, indicariam um percentual de tempo gasto que ultrapassaria o tempo preconizado nas citadas Resoluções.

Outro ponto importante a que chegamos neste estudo refere-se ao indicativo da necessidade de prosseguir estudando o assunto - dimensionamento de pessoal de enfermagem, no sentido de desvelar suas outras variáveis como, por exemplo, os escores definidos em sistemas de classificação de pacientes por intervenção terapêutica que vêm sendo estudados e utilizados em Unidades de Terapia Intensiva; além das ausências previstas e não previstas para se estabelecer os índices de segurança técnica, compatíveis com cada realidade, conforme alertam estudiosas do assunto ${ }^{(15)}$.

Finalizando, consideramos que, apesar de suas limitações, o presente estudo constituiu-se em indicativo importante para outros trabalhos a respeito da temática - dimensionamento de pessoal de enfermagem, pelo fato de explicitar a metodologia de mensuração de tempo e movimento que foi utilizada.

Apreendemos ainda, a necessidade de prosseguir com outros estudos que abarquem, também, a dimensão da saúde dos trabalhadores de enfermagem, compreendida em sua abrangência e sentido mais amplo, que engloba a qualidade de vida e humanização do trabalho, aspectos esses, em geral não contemplados em estudos que utilizam métodos puramente quantitativos, para o dimensionamento de pessoal, como este.

\section{REFERÊNCIAS}

1. Germano RM. Educação e ideologia da enfermagem no Brasil. São Paulo: Cortez; 1983. cap. 1. p. 39-40.

2. Ide CC. Prática de enfermagem em UTI e contexto de saúde. Rev Esc Enferm USP. 1989;23(1):91-8.

3. Car MR. Problemas de enfermagem da esfera física em pacientes hospitalizados: caracterização por unidades de internação, cuidado semi-intensivo e tratamento intensivo [dissertação]. São Paulo: Escola de Enfermagem, Universidade de São Paulo; 1986.

4. Gaidzinsk RR. Dimensionamento de pessoal de enfermagem em instituições hospitalares [tese livre-docência]. São Paulo: Escola de Enfermagem, Universidade de São Paulo; 1998.

5. Conselho Federal de Enfermagem (COFEN). Resolução nº 189/ 96. Estabelece parâmetros para dimensionamento do quadro de profissionais de enfermagem nas instituições de saúde. In: Conselho Regional de Enfermagem de São Paulo. Documentos básicos de enfermagem: enfermeiros, técnicos e auxiliares. São Paulo; 2001. p.144-51.

6. Conselho Federal de Enfermagem (COFEN). Resolução n ${ }^{2}$ 293/ 2004. Fixa e estabelece parâmetros para o dimensionamento de pessoal de enfermagem nas unidades assistenciais das instituições de saúde e assemelhados. In: Conselho Regional de Enfermagem de São Paulo [legislação na Internet]. São Paulo; 2004. [citado 2005 jun. 15]. Disponível em: http://www.corensp. org.br/resoluções/Resolucao293.htm

7. Fugulim FMT. Dimensionamento de pessoal de enfermagem: avaliação do quadro de pessoal das unidades de internação de um hospital de ensino [tese]. São Paulo: Escola de Enfermagem, Universidade de São Paulo; 2002.
8. Ricardo CM, Fugulin FMT, Souza TM. Dimensionamento de pessoal de enfermagem: análise do tempo efetivo de trabalho das enfermeiras da UTI pediátrica do HU-USP. Rev Gaúcha Enferm. 2004;25(3):357-66.

9. Lunardi VL, Lunardi Filho WD, Borba MR. Como a enfermeira utiliza o tempo de trabalho numa unidade de internação. Rev Bras Enferm. 1994;7(1):7-14.

10. Prescott PA, Phillips CY, Ryan JN, Thompson KO. How nurses spend their time. Image J Nurs Sch. 1991;23(1):23-8.

11.Mello MC. Estudo do tempo no trabalho da enfermagem: construção de instrumento de classificação de atividades para implantação do método amostragem do trabalho [dissertação]. São Paulo: Escola de Enfermagem, Universidade de São Paulo; 2002.

12. Rodrigues Filho J. Sistema de classificação de pacientes. Parte I: dimensionamento de pessoal de enfermagem. Rev Esc Enferm USP. 1992;26(3):395-404.

13. Giovanetti P. Understanding patient classification systems. J Nurs Adm. 1979;9(2):4-9.

14. Gaidzinski RR. O dimensionamento do pessoal de enfermagem segundo a percepção de enfermeiros que vivenciam essa prática [tese]. São Paulo: Escola de Enfermagem, Universidade de São Paulo; 1994.

15. Fugulin FMT, Gaidzink RR, Kurcgant P. Ausências previstas e não previstas da equipe de enfermagem das unidades de internação do HU-USP. Rev Esc Enferm USP. 2003;37(4): 109-17. 